\title{
342.
}

\section{ON THE CONICS WHICH PASS THROUGH THREE GIVEN POINTS AND TOUCH A GIVEN LINE.}

[From the Quarterly Journal of Pure and Applied Mathematics, vol. vi. (1864), pp. $24-30$.

CONSIDER the system of conics which pass through three given points and touch a given line; if among these we select the conics which touch an assumed line, it is easy to show analytically that there are four such conics, all real or else all imaginary; viz. the three points form a triangle, and if the two lines cut the three sides produced or cut the same two sides and the third side produced, then the conics are all real; but in every other case they are all imaginary. The latter part of the theorem may also be seen geometrically; in fact, if a triangle is inscribed in a conic, say first in an ellipse, or in a parabola, or in one branch of a hyperbola, then all the tangents of the conic (and therefore any two tangents whatever) cut the three sides produced, but if the triangle is inscribed in the two branches of a hyperbola (that is, two vertices on one branch and the remaining vertex on the other branch), then all the tangents of the conic (and therefore any two tangents whatever) cut the same two sides and the third side produced: and thus the only real conics are those which cut the three sides produced, or else the same two sides and the third side produced. The analytical proof referred to is as follows: taking $(x=0, y=0, z=0)$ for the equations of the sides of the triangle, the equation of a conic through the three points is

$$
\frac{f}{x}+\frac{g}{y}+\frac{h}{z}=0
$$

or, what is the same thing,

$$
2 f y z+2 g z x+2 h x y=0
$$

that is

$$
(0,0,0, f, g, h \gamma x, y, z)^{2}=0 \text {. }
$$


The inverse coefficients are

$$
\left(-f^{2},-g^{2},-h^{2}, \quad g h, \quad h f, \quad f g\right),
$$

and hence the condition in order that the conic may touch the line $\alpha x+\beta y+\gamma z=0$ is

$$
\left(f^{2}, \quad g^{2}, \quad h^{2},-g h,-h f,-f g \gamma \alpha, \beta, \gamma\right)^{2}=0,
$$

or, what is the same thing,

$$
\sqrt{ }(\alpha f)+\sqrt{ }(\beta g)+\sqrt{ }(\gamma h)=0 .
$$

Similarly the condition in order that the conic may touch the line $l x+m y+n z=0$ is $\sqrt{ }(l f)+\sqrt{ }(m g)+\sqrt{ }(n h)=0$. Hence if the conic touch the two lines, we have

$$
\sqrt{ }(f): \sqrt{ }(g): \sqrt{ }(h)=\sqrt{ }(\beta n)-\sqrt{ }(\gamma m) \quad: \sqrt{ }(\gamma l)-\sqrt{ }(\alpha n) \quad: \sqrt{ }(\alpha m)-\sqrt{ }(\beta l),
$$

or, what is the same thing,

$$
f: g: \quad h=\beta n+\gamma m-2 \sqrt{ }(\beta \gamma m n): \gamma l+\alpha n-2 \sqrt{ }(\gamma \alpha n l): \alpha m+\beta l-2 \sqrt{ }(\alpha \beta l m),
$$

which, since the radicals must be so taken that the product may be $=\alpha \beta \gamma l m n$, gives in all four conics: and these will be all real if the signs of $(l, m, n)$ are the same with, or opposite to those of $(\alpha, \beta, \gamma)$ respectively; which proves the theorem.

In particular since infinity is a line meeting the three sides produced; if the given line meet the three sides produced, the system will contain four real parabolas; but, if the given line meets two sides and a side produced, there is not any real parabola. In the latter case, as is obvious geometrically, the conics of the system are all hyperbolas.

Any side of the triangle, and the line joining the opposite vertex with the point of intersection of the side and given line, form a pair of lines passing through the three points and meeting on the given line; such pair of lines is a conic of the system; and we have thus three pairs of lines, each pair a conic of the system.

We may by what precedes form some idea of the nature of the system of conics which pass through the three given points and touch the given line. In fact writing at the point of contact the letters $H, P, E, L$ according as the conic is a hyperbola, parabola, ellipse, or pair of lines, then if the given line cut the three sides produced, we have as in fig. 1 .

FIG. 1.

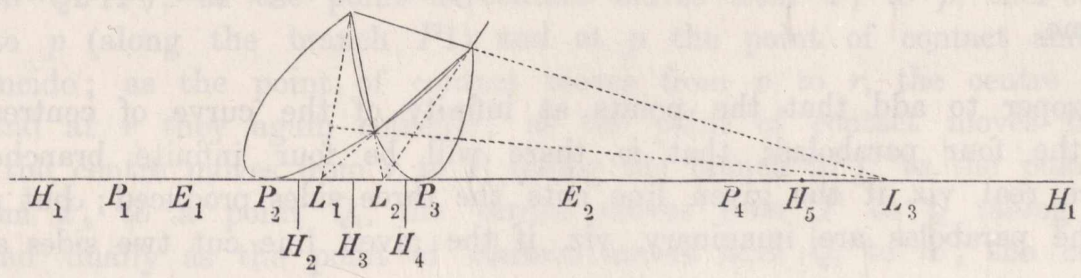


Whereas, if the given line cuts two sides and a side produced, we have more simply as in fig. 2.

FIg. 2.

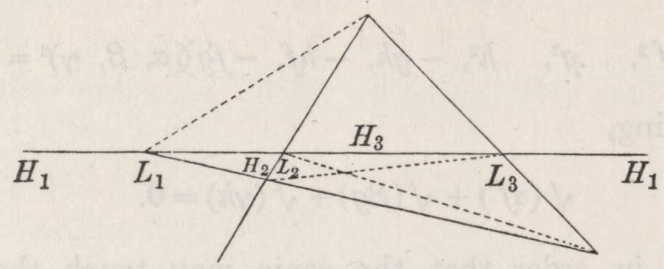

But to gain a more precise knowledge, it is proper to consider the curve which is the locus of the centres of the conics of the system.

Such locus which, as will presently be seen, is a curve of the fourth order, must, it is clear, pass through the points of intersection $\left(L_{1}, L_{2}, L_{3}\right.$ in figs. 1 and 2 respectively and $p, q, r$ in fig. 3 presently referred to) of the sides with the given line; and it is not difficult to show geometrically that it touches, at these points, the sides of the triangle. It may be shown also that the curve has three nodes (double points), viz. the middle point of each side of the triangle is a node of the curve. In fact if upon any side as base we apply an equal and opposite triangle so as to form with the given triangle a parallelogram, then any conic through the four vertices of the parallelogram will have for its centre the central point of the parallelogram; that is, the middle point of the side in question. But we may through the four vertices describe two conics, each of them touching the given line; that is the middle point of the side is the centre of two different conics of the system, and it is therefore a node upon the curve of centres. And moreover the node will be a crunode or an acnode (i.e. a double point with two real branches, or else a conjugate or isolated point) according as the conics are real or imaginary: and it is easy to see that if the given line does not cut the parallelogram, or if it cuts two opposite sides, the conics will be both real; but if it cuts two adjacent sides the conics will be both imaginary; that is, in the former case we have a crunode, and in the latter an acnode. Through each node may be drawn two tangents to the curve; and it is a known property of curves of the fourth order that the six points of contact lie on a conic; one of the tangents through the node is however the side whereon the node lies, and the points of contact of the three sides lie on a line, viz. the given line: hence the last mentioned conic is composed of the given line, and another line; that is, the three points of contact of the other tangents through the three nodes lie on this other line.

It is proper to add that the points at infinity of the curve of centres are the centres of the four parabolas; that is, there will be four infinite branches, if the parabolas are real, viz. if the given line cuts the three sides produced; but no infinite branch if the parabolas are imaginary, viz. if the given line cut two sides and a side produced. 
The triangle and the three triangles applied to the three sides form together a triangle similar to the original triangle but of double the linear magnitude, and the form of the curve of centres depends as has been shown on the position of the given line in regard to the triangle and the double triangle. The cases to be considered are tolerably numerous, but it is easy from the foregoing considerations, to see in any particular case what is the form of the curve of centres; for facility of delineation I select a form without infinite branches, see fig. 3 , in which the given line cuts the two sides $C A, C B$, and the third side $A B$ produced; it is moreover to be observed

FIG. 3.

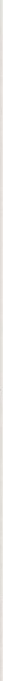

that as the figure is drawn the given line cuts the two sides $C A, C B$ below their middle points $Q$ and $P$ respectively. By what precedes it appears that the middle points $Q, P$ of these two sides $C A, C B$ are each of them crunodes, but that the middle point $R$ of the remaining side $A B$ is an acnode. And this being so the general form of the curve is at once perceived to be that shown by fig. 3 .

It is very interesting to trace the corresponding positions of the point of contact on the given line, and of the centre on the curves of centres. When the point of contact is at $\infty$, the centre is at $I$, as the point of contact moves from $\infty$ to $q$, the centre moves from $I$ to $q$, and at $q$ the two coincide; as the point of contact moves from $q$ to a point $Q_{2}$, the centre moves from $q$ to $Q$ (along the branch $Q 2$ ); as the point of contact moves from $Q_{2}$ to a point $P_{1}$, the centre moves from $Q$ to $P$ (along the branch $Q 21 P$ ); as the point of contact moves from $P_{1}$ to $p$, the centre moves from $P$ to $p$ (along the branch $P 1$ ) and at $p$ the point of contact and the centre again coincide; as the point of contact moves from $p$ to $r$, the centre moves from $p$ to $r$ and at $r$ they again coincide; as the point of contact moves from $r$ to a point $P_{2}$ the centre moves from $r$ to $P$ (along the branch $2 P$ ); as the point of contact moves from $P_{2}$ to a point $Q_{1}$, the centre moves from $P$ to $Q$ (along the branch $P 21 Q$ ) and finally as the point of contact moves from $Q_{1}$ to $\infty$, the centre moves from $Q$ (along the branch $Q_{1}$ ) to $I$, thus completing the circuit. 
The equation of the curve of centres was given in the late $\mathrm{Mr}$ Hearn's "Researches on Curves of the Second Order, \&c. London, 1846," viz. if $x=0, y=0, z=0$ be the equations of the sides of the triangle formed by the given points; $x+y+z=0$ the equation of the line infinity, and $\alpha x+\beta y+\gamma z=0$ the equation of the given line, then the equation of the curve of centres is

$$
\sqrt{ }\{\alpha x(-x+y+z)\}+\sqrt{ }\{\beta y(x-y+z)\}+\sqrt{ }\{\gamma z(x+y-z)\}=0,
$$

or more generally if $x+y+z=0$ be the equation of an assumed line, then this equation is that of the locus of the pole of the assumed line in regard to the conics passing through the given points and touching the given line, see my paper "Note on a Family of Curves of the Fourth Order," Cambridge and Dublin Mathematical Journal, t. v. (18こ0), pp. 148-152, [85], where I have noticed the above mentioned property, that the conic through the points of contact of the tangents through the nodes breaks up into a pair of lines. It is I think worth while to show how the equation is obtained. The equation of a conic through the given points and touching the given line is

$$
\left(0,0,0, f, g, h \gamma(x, y, z)^{2}=0\right.
$$

with the condition $\sqrt{ }(x f)+\sqrt{ }(\beta g)+\sqrt{ }(\gamma h)=0$, and this being so, the coordinates of the pole in relation thereto, of the assumed line $x+y+z=0$, are

We have thence

$$
\begin{aligned}
x: y: z= & (-f+g+h) f \\
: & (f-g+h) g \\
: & (f+g-h) h .
\end{aligned}
$$

$$
\begin{aligned}
-x+y+z \text { proportional to } & -(-f+g+h) f \\
& +(f-g+h) g \\
& +(f+g-h) h,
\end{aligned}
$$

that is, to $f^{2}-(g-h)^{2}$, which is $=(f-g+h)(f+g-h)$,

and combining with this the equation

we obtain

$$
\alpha x \text { proportional to }(-f+g+h) f \alpha,
$$

that is

$$
\boldsymbol{\alpha} x(-x+y+z) \text { proportional to } \alpha f \text {, }
$$

$$
\alpha x(-x+y+z): \beta y(x-y+z): \gamma z(x+y-z)=\alpha f: \beta g: \gamma h,
$$

so that from the equation $\sqrt{ }(\alpha f)+\sqrt{ }(\beta g)+\sqrt{ }(\gamma h)=0$, we have at once the foregoing equation

$$
\sqrt{ }\{\boldsymbol{\alpha} x(-x+y+z)\}+\sqrt{ }\{\beta y(x-y+z)\}+\sqrt{ }\{\boldsymbol{\gamma} z(x+y-z)\}=0 .
$$

The rationalised form is

$$
\left(1,1,1,-1,-1,-1 \gamma(\alpha x(-x+y+z), \quad \beta y(x-y+z), \quad \gamma z(x+y-z))^{2}=0,\right.
$$


which shows what has been all along assumed, that the curve is of the fourth order. This equation may be transformed into

$$
\begin{aligned}
& x^{2}\left(\alpha^{2} x^{2}+\beta^{2} y^{2}+\gamma^{2} z^{2}-2 \beta \gamma y z+2 \gamma \alpha z x+2 \alpha \beta x y\right) \\
+\quad & y^{2}\left(\alpha^{2} x^{2}+\beta^{2} y^{2}+\gamma^{2} z^{2}+2 \beta \gamma y z-2 \gamma \alpha z x+2 \alpha \beta x y\right) \\
+ & z^{2}\left(\alpha^{2} x^{2}+\beta^{2} y^{2}+\gamma^{2} z^{2}+2 \beta \gamma y z+2 \gamma \alpha z x-2 \alpha \beta x y\right) \\
- & 2 y z(\alpha x+\beta y+\gamma z)(-\alpha x+\beta y+\gamma z) \\
- & 2 z x(\alpha x+\beta y+\gamma z)(\alpha x-\beta y+\gamma z) \\
- & 2 x y(\alpha x+\beta y+\gamma z)(\alpha x+\beta y-\gamma z)=0:
\end{aligned}
$$

if with this equation we combine the equation $\alpha x+\beta y+\gamma z=0$, we find at the points of intersection with the given line

that is

$$
x^{2} .4 \beta \gamma y z+y^{2} \cdot 4 \gamma \alpha z x+z^{2} .4 \alpha \beta x y=0,
$$

$$
x y z(\beta \gamma x+\gamma \alpha y+\alpha \beta z)=0,
$$

so that the points in question are the intersections of the given line $\alpha x+\beta y+\gamma z=0$, with the lines $x=0, y=0, z=0, \frac{x}{\alpha}+\frac{y}{\beta}+\frac{z}{\gamma}=0$. The point $\alpha x+\beta y+\gamma z=0, \frac{x}{\alpha}+\frac{y}{\beta}+\frac{z}{\gamma}=0$ corresponds to the conic which touches the given line at its intersection with the assumed line $x+y+z=0$, the pole in relation to this conic is obviously a point on the given line. The point in question, if $x+y+z=0$ denote the line infinity, is the point $I$ of fig. 3.

It may be proper to mention a far less symmetrical form of the equation of the conic, but which has the advantage of putting in evidence the point of contact; viz. the equation is expressed in terms of the parameter $\alpha$ denoting the distance of the point of contact from a given point in the base line, and which is therefore very convenient for tracing the changes of form of the conic. Assuming as before that the base line cuts the sides produced, then (see fig. 4) if of the three points 1 denote

FIG. 4 .

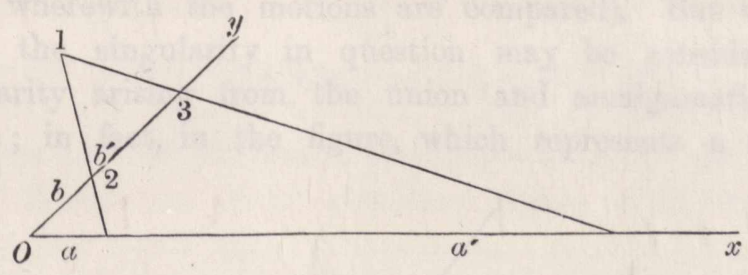

that which is furthest from, and 2 that which is nearest to the base line, and if the base line be taken as the axis of $x$, and 23 as the axis of $y$; the equation of the base line is $y=0$, and the equations of the sides $23,31,12$ are $x=0, \frac{x}{a^{\prime}}+\frac{y}{b^{\prime}}=1$, $\frac{x}{a}+\frac{y}{b}=1$, where $a, b, a^{\prime}, b^{\prime}, a^{\prime}-a, b^{\prime}-b, a^{\prime} b-a b^{\prime}$ are all positive, so that, by choosing 
the axes as above, we avoid the consideration of the several cases corresponding to different signs of these quantities. And this being so, if $x=\alpha$ is the coordinate of the point of contact, the equation of the conic is

where

$$
\left(A, B, C, F, G, H \gamma(x, y, 1)^{2}=0\right. \text {, }
$$

and these give

$$
\begin{aligned}
& A=2 b b^{\prime}\left(a^{\prime}-a\right), \\
& B=2 \alpha^{2} \quad\left(a^{\prime}-a\right), \\
& C=2 \alpha^{2} b b^{\prime}\left(a^{\prime}-a\right) \\
& F=-\alpha^{2} \quad\left(a^{\prime}-a\right)\left(b^{\prime}+b\right), \\
& G=-2 a b b^{\prime}\left(a^{\prime}-a\right) \\
& H=\left\{\left(\alpha^{2}+a a^{\prime}\right)\left(b^{\prime}-b\right)-2 \alpha\left(a b^{\prime}-a^{\prime} b\right)\right\}
\end{aligned}
$$

$$
\begin{aligned}
& A B-H^{2}=- {\left[\left\{(\alpha-a) \sqrt{ }\left(b^{\prime}\right)+\left(\alpha-a^{\prime}\right) \sqrt{ }(b)\right\}^{2}-\left(a^{\prime}-a\right)\left(a^{\prime} b-a b^{\prime}\right)\right] \times } \\
& \quad\left[\left\{(\alpha-a) \sqrt{ }\left(b^{\prime}\right)-\left(\alpha-a^{\prime}\right) \sqrt{ }(b)\right\}^{2}-\left(a^{\prime}-a\right)\left(a^{\prime} b-a b^{\prime}\right)\right], \\
& B C-F^{2}=-a^{4}\left(a^{\prime}-a\right)^{2}\left(b^{\prime}-b\right)^{2} \\
& C A-G^{2}= 0 \\
& G H-A F=-2 b b^{\prime}\left(a^{\prime}-a\right)\left(b^{\prime}-b\right) \alpha(\alpha-a)\left(\alpha-a^{\prime}\right) \\
& H F-B G=-\left(a^{\prime}-a\right)\left(b^{\prime}-b\right) \alpha^{2}\left\{\left(b^{\prime}+b\right)\left(\alpha^{2}+a a^{\prime}\right)-2 \alpha\left(a b^{\prime}+a^{\prime} b\right)\right\} \\
& F G-C H=-2 b b^{\prime}\left(a^{\prime}-a\right)\left(b^{\prime}-b\right) \alpha^{2}(\alpha-a)\left(\alpha-a^{\prime}\right) .
\end{aligned}
$$

The condition that the conic may be a parabola is $A B-H^{2}=0$, which gives, as it should do, four real values of $\alpha$.

2, Stone Buildings, W.C. 